\title{
Resveratrol reduces COMPopathy in mice through activation of autophagy
}

Jacqueline T. Hecht ${ }^{1}{ }^{2}$, Francoise Coustry ${ }^{1}$, Alka C. Veerisetty ${ }^{1}$, Mohammad G. Hossain and Karen L. Posey ${ }^{1}$

Department of Pediatrics McGovern Medical School at The University of Texas Health Science Center at Houston (UTHealth), TX1 and UTHealth School of Dentistry².

Running title: Resveratrol induced autophagy clears mutant-COMP

To whom correspondence should be sent:

Karen L. Posey, PhD

Department of Pediatrics

McGovern Medical School at The University of Texas Health Science Center at Houston (UTHealth)

6431 Fannin

Houston, TX 77030

Phone: (713) 500-5786

Fax: (713) 500-5689

Email: Karen.Posey@uth.tmc.edu

ORCID: https://orcid.org/0000-0003-1234-6087

Study design: KP and JH. Data collection: FC, MH and AV. Data analysis: FC, KP and JH. Data interpretation: FC, KP and $\mathrm{JH}$. Drafting manuscript: $\mathrm{KP}$ and $\mathrm{JH}$. Revising manuscript content: $\mathrm{KP}$ and $\mathrm{JH}$. Approving final version of manuscript: $\mathrm{JH}, \mathrm{FC}, \mathrm{MH}, \mathrm{AV}$ and $\mathrm{KP}$. $\mathrm{KP}$ and $\mathrm{JH}$ takes responsibility for the integrity of the data analysis. The authors have no conflict of interest to declare. 


\section{Abstract}

Misfolding mutations in cartilage oligomeric matrix protein (COMP) cause it to be retained within in ER of chondrocytes, stimulating a multitude of damaging cellular responses including ER stress, inflammation and oxidative stress which ultimately culminates in the death of growth plate chondrocytes and pseudoachondroplasia (PSACH). Previously, we demonstrated that an antioxidant, resveratrol, substantially reduces the intracellular accumulation of mutant COMP, dampens cellular stress and lowers the level of growth plate chondrocyte death. In addition, we showed that resveratrol reduces mTORC1 (mammalian target of rapamycin complex 1) signaling, suggesting a potential mechanism. In this work, we investigate the role of autophagy in treatment of COMPopathies. In cultured chondrocytes expressing wild type or mutant COMP (MT-COMP), resveratrol significantly increased the number of large LC3 vesicles, directly demonstrating that resveratrol stimulated autophagy is an important component of the resveratrol-driven mechanism responsible for the degradation of mutant COMP. Moreover, pharmacological inhibitors of autophagy suppressed degradation of MT-COMP in our established mouse model of PSACH. In contrast, blockage of the proteasome did not substantially alter resveratrol clearance of mutant COMP from growth plate chondrocytes. Mechanistically, resveratrol increased SIRT1 and PP2A expression and reduced MID1 expression and activation of pAKT and mTORC1 signaling in growth plate chondrocytes, allowing clearance of mutant COMP by autophagy. Importantly, we show that optimal reduction in growth plate pathology, including decreased mutant COMP retention, decreased mTORC1 signaling and restoration of chondrocyte proliferation was attained when treatment was initiated between birth to one week of age in MT-COMP mice, translating to birth to approximately 2 years of age in PSACH children. These results clearly demonstrate that resveratrol stimulates clearance of mutant COMP by an autophagy-centric mechanism.

Key Words: pseudoachondroplasia, autophagy, resveratrol, dwarfism, cartilage oligomeric matrix protein (COMP), proteasome, mTORC1 
Keywords: Genetic animal models, Preclinical Studies, Growth plate, Therapeutics, Diseases and Disorders of/related to Bone

\section{Introduction}

Next generation sequencing has resulted in a huge expansion of information that has led to greater understanding of the mutational underpinnings of genetic disorders ${ }^{(1)}$. This is particularly true for skeletal dysplasias, in which 425 distinct disorders are caused by mutations in 437 different genes $^{(1)}$. Mouse modeling of the genetic mutations generating specific skeletal dysplasia has revealed the mechanistic pathways that underlie the resulting pathologies, thereby identifying therapeutic targets. Previously, we showed that mutations in cartilage oligomeric matrix protein (COMP) causes pseudoachondroplasia (PSACH; COMPopathy), a well-recognized and clinically characterized severe dwarfing condition(2). The MT-COMP transgenic mouse, with the common D469del mutation that recapitulates the short stature and skeletal finding of PSACH, was used to define the pathology that specifically affects growth plate chondrocytes ${ }^{(3-7)}$. In this study, mechanisticdriven therapeutic approaches in the MT-COMP mouse were used to define the mechanisms of resveratrol therapy.

Chondrocytes are secretory cells that generate abundant extracellular matrix proteins necessary to build cartilage. COMP is normally synthesized in the rER cisternae and is processed through the Golgi then exported to the ECM where it forms an ordered matrix network ${ }^{(8)}$. It has long been known that mutant protein accumulates in PSACH chondrocytes $^{(9-11)}$, mutations in COMP were shown to cause PSACH ${ }^{(10,11)}$. It was subsequently shown that mutant COMP misfolds in the ER and prematurely assembles an ordered matrix involving types 2 and 9 collagens, matrilin 3 and other extracellular proteins, resulting in massive intracellular protein accumulation ${ }^{(7,12,13)}$. The protein accumulation in the ER of chondrocytes initiates a self-perpetuating pathological loop between oxidative stress, inflammation and ER stress that leads to elevated mTORC1 signaling, DNA damage and death of chondrocytes ${ }^{(4-6,14)}$. Misfolded proteins in the ER are typically degraded through 
macroautophagy (henceforth referred to as autophagy) or by the proteasome after being translocated out of the $\mathrm{ER}^{(15)}$. In the presence of mutant COMP, the high volume of stalled protein/mis-folded protein/intracellular matrix either overwhelms the cellular clearance mechanisms and/or blocks export and degradation. In either situation, these clearance mechanisms do not efficiently clear mutant COMP from the ER and normal chondrocyte function is disrupted.

Previously, we demonstrated that treatment of MT-COMP mice with resveratrol or aspirin from birth to 4 weeks decreased mutant COMP intracellular retention, inflammation, chondrocyte cell death and restored chondrocyte proliferation $(3,4,7,16)$. Notably, these treatments rescued $50 \%$ of the loss of femur length. These studies now define the cellular processes stimulated by resveratrol (aspirin) that prevent mutant COMP accumulation and, importantly, define the appropriate timing of treatment.

\section{Material and Methods}

Bigenic mice

The MT-COMP (C57BL/6 mus musculus) mice used in these and previously described experiments contain the pTRE-COMP (coding sequence of human COMP+FLAG tag driven by the tetracycline responsive element promoter) and pTET-On-Col II (rtTA coding sequence driven by a type II collagen promoter)(17-19). The presence of these two transgenes had not led to any readily detectable health concerns. Male and female mice were administered DOX (500 ng/ml) postnatally in their drinking water until collection. Mice were housed as a single sex group after weaning and fed standard chow (PicoLab rodent diet 20 \#5053). Ten mice were in each treatment group or control group and litters were assigned to each group until each group contained at least 10 animals. Investigators were not blinded during allocation and animal handling but were blinded at analysis. These studies were approved by the UTHealth Animal Welfare Committee.

\section{Immunohistochemistry}

Hind limbs from male MT-COMP and C57BLI6 control mice were collected and tibial growth plates analyzed as previously described ${ }^{(52)}$. The limbs were fixed in $95 \%$ ethanol and pepsin 
$(1 \mathrm{mg} / \mathrm{ml}$ in $0.1 \mathrm{~N} \mathrm{HCl})$ was used for antigen retrieval. Immunostaining was performed by incubating different sections overnight at $4^{\circ} \mathrm{C}$ using the following antibodies: human COMP (Abcam Cambridge, MA ab11056-rat 1:100), pS6 (1:200 2215S rabbit polyclonal Cell Signaling Technology), PI3K-1 (abcam- ab 225720-1:200), SIRT-1 (abcam: ab 32441 - 1:100), pAMPK (Invitrogen: PA537821- 1:100), interleukin 16 (IL-16) (Santa Cruz Biotechnology; sc-7902, 1:100), YM1/ECF-L (Stem Cell Technologies; 01404, 1:100) and PCNA (abcam ab92552 1:200). Species specific biotinylated secondary antibodies were used for $1 \mathrm{hr}$ at RT. Sections were then washed and incubated with streptavidin horseradish peroxidase (HRP) and DAB was used as chromogen. The sections were dehydrated and mounted with Thermofisher cytoseal 60 and then visualized under the BX51 inverted microscope (Olympus America, Center Valley, PA). Limbs were fixed in $10 \% \mathrm{wt} /$ vol formalin for terminal deoxynucleotidyl transferase-mediated deoxyuridine triphosphate-biotin nick end labeling (TUNEL) staining. A priori data indicate that the percentage TUNEL positive chondrocytes are approximately $80 \%$ in untreated mice and $20 \%$ in mice treated with resveratrol from birth to 4 weeks, with within group standard deviations of around $12 \%$. For a one-way ANOVA analysis with post-hoc Tukey test to identify difference in TUNEL percentages between 5 groups at a Type I error rate of 0.05 , a sample size of 10 mice per group would have $80 \%$ power to identify an effect size (Cohen's d) of 0.51 . This would translate into pair-wise differences in means of at least $17 \%$ in at least two of the 5 groups.

\section{Drug treatments}

No adverse events were associated with these drugs administered as described. Rapamycin treated $2 \mathrm{mg} / \mathrm{kg}$ reduced viability of pups and the dosage was reduced to $1 \mathrm{mg} / \mathrm{kg}$.

Resveratrol: Resveratrol (Reserveages Organics liquid resveratrol super berry extract), with known antioxidant/anti-inflammatory properties ${ }^{(20-22)}$, was administered in a dose of $0.25 \mathrm{~g} / \mathrm{L}$ in the drinking water ${ }^{(3)}$ starting either from birth (transmitted through maternal breast milk) or 1 or 2 or 3 weeks and continued until 4 weeks of age ${ }^{(3)}$. 
Aspirin: Aspirin, a COX2 inhibitor that reduces inflammation was administered in the drinking water in a dose of $0.3 \mathrm{~g} / \mathrm{L}$ of aspirin (Sigma, USA) from birth to 4 weeks ${ }^{(3)}$.

Rapamycin: Rapamycin (Tocris Bioscience, United Kingdom) inhibits mTOR pathway, inducing autophagy ${ }^{(23)}$, was dissolved in ethanol at $1 \mathrm{mg} / \mathrm{ml}$ and stored in $-20^{\circ} \mathrm{C}$. On the day of injection, rapamycin was diluted with $\mathrm{PBS}$ to $0.1 \mathrm{mg} / \mathrm{ml}$. Intraperitoneal injection of rapamycin at $1 \mathrm{mg} / \mathrm{kg}$ was performed 5 days per week from 1 - 4 weeks of age ${ }^{(24)}$. .

Metformin: Metformin (Sigma, USA) activates AMPK, which in turn represses mTORC1 activity stimulating autophagy ${ }^{(25,26)}$, was administered at $0.5 \mathrm{mg} / \mathrm{ml}$ in DOX drinking water from birth (through maternal breast milk) to 4 weeks ${ }^{(24,27)}$.

Bafilomycin A: Bafilomycin A (Cayman Chemical, USA) blocks autophagy by preventing fusion of autophagosome with lysosome ${ }^{(28)}$ was dissolved in $10 \mathrm{mg} / \mathrm{ml} \mathrm{DMSO}$, aliquoted and stored at $20^{\circ} \mathrm{C}$. For each subcutaneous injection, bafilomycin A was freshly diluted to $0.05 \mathrm{mg} / \mathrm{ml}$ in PBS. Mice were injected with $0.3 \mathrm{mg} / \mathrm{Kg}$ of bortezomib 5 times per week beginning at age 1 week $^{(29)}$. Bafilomycin A administration to untreated MT-COMP did not alter mutant COMP accumulation.

Bortezomib: Bortezomib (\# 2164 AvaChem Scientific, USA) inhibits the chymotryptic activity of the proteasome (20S subunit) reducing proteasomal degradation ${ }^{(30)}$ was dissolved in $10 \mathrm{mg} / 1 \mathrm{ml}$ DMSO, aliquoted and stored at $-20^{\circ} \mathrm{C}$. Freshly diluted bortezomib was diluted for each subcutaneous injection to $0.01 \mathrm{mg} / \mathrm{ml}$ in PBS. Mice were injected with $0.1 \mathrm{mg} / \mathrm{Kg}$ of bortezomib 3 times per week beginning at age 1 week $^{(31,32)}$. Bortezomib administration to untreated MT-COMP did not alter mutant COMP accumulation. 


\section{Cell culture}

RCS cells expressing either human wild-type or MT-COMP were generated using the Lenti-XTet-On advanced inducible expression system according to the manufacturer protocol (Takara Bio Company, Mountain View, CA). Briefly, RCS cells were infected with high-titer lentiviral preparations of pLVX-Tet-on advanced vector or pLVX-Tight-human D469del-MT-COMP vector; and selected with puromycin and G418. Expression of human COMP in RCS cells was validated by Western Blot with anti-Flag antibody to recognize the tagged COMP (1:5000 F7425 rabbit polyclonal from Sigma, Saint Louis, MO). DOX was used for 4 days to induce COMP expression in RCS cells, which were then treated for 1 day as follows: 1) control with no chloroquine and no resveratrol, 2) chloroquine $(50 \mu \mathrm{M}), 3)$ resveratrol $(50 \mu \mathrm{M})$ and 4) both resveratrol $(50 \mu \mathrm{M})$ and chloroquine $(50 \mu \mathrm{M})$. The cells were fixed post-treatment with methanol at $-20^{\circ} \mathrm{C}$ for 10 minutes, rinsed with PBS and stored at $4^{\circ} \mathrm{C}$. Coverslips were then incubated for $1 \mathrm{~h}$ at $37^{\circ} \mathrm{C}$ with LC3 primary antibody (Cell signaling; 2775; 1:500). Primary antibodies were detected using Alexa Fluor 594 for $1 \mathrm{~h}$ at $37^{\circ} \mathrm{C}$ (dilution 1:1000). Images were collected with confocal microscope at 63x oil immersion lens (Zeiss LSM-510). A priori data suggests that the median number of foci per image for each of the 8 groups would range from 0 through 2 with interquartile ranges of 0 through 3. Assuming a power of 0.80 and a Type I error rate of 0.05 , a sample size of 23 cells counted per image will provide us with sufficient power to identify a small effect size of 0.1 (Cohen's d). For our analysis, we elected to count 30 to 50 cells per image. ImageJ was used to count LC3 foci on ten images for each group with 30-50 cells per image. T-tests were used to compare each group to the other groups and scorers were blinded.

\section{Results}

Resveratrol treatment reactivates autophagy in MT-COMP chondrocytes

Autophagy and proteasomal degradation were examined as they are the cellular mechanisms responsible for removing misfolded protein from the $\mathrm{ER}^{(33)}$. Previously, we showed that MT- 
COMP accumulation in the ER of murine MT-COMP growth plate chondrocytes occurs because autophagy was blocked by elevated mTORC1 signaling(4). It remains unclear whether proteasome degradation plays a role in this ER-stress-induced chondrocyte pathology because COMP-ECM matrix within the ER may prohibit translocation from the ER to the proteasome ${ }^{(4)}$. To determine whether autophagy and/or the proteasomal degradation were responsible for resveratrol clearance of MT-COMP, neonatal mice were treated with resveratrol birth to 4 weeks in the absence or presence of an autophagy blocker (bafilomycin $\mathrm{A}^{(28)}$ ) or a proteasomal inhibitor (bortezomib $^{(30)}$ ) from 1 to 4 weeks of age. As shown in Figure 1 Panel 1B, resveratrol treatment alone dramatically reduced MT-COMP accumulation compared to untreated growth plate chondrocytes (Fig. 1 Panel 1A) ${ }^{(3)}$. The addition of bafilomycin A largely prevented resveratrolstimulated clearance of MT-COMP (Fig. 1 Panel 1C) while bortezomib only partially reduced MTCOMP clearance (Fig. 1 Panel 1D). This shows that autophagy is the primary mechanism of resveratrol stimulated MT-COMP clearance and proteasome activity plays a minor role.

To further demonstrate that resveratrol stimulates autophagy in chondrocytes, resveratrolinduced autophagic vesicle clearance was pharmacologically blocked using chloroquine to allow visualization of autophagic vesicles, since they are rapidly degraded under normal conditions. Rat chondrosarcoma (RCS) cells expressing either wild type (WT) or mutant COMP were treated with resveratrol in presence or absence of chloroquine and the number of microtubule-associated protein light chain 3 (LC3) positive vesicles (foci), the classic marker of autophagy, were

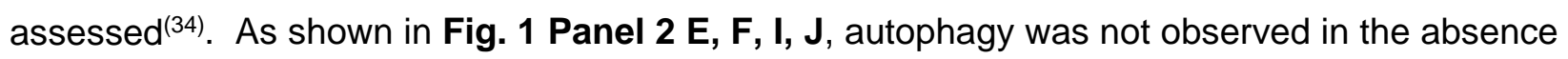
of chloroquine, as expected. The number of autophagic vesicles was significantly increased with resveratrol treatment (Fig. 1 Panel 2 H, L, M) over the basal level of autophagy $(\mathbf{G}, \mathbf{K})$ confirming that resveratrol stimulates autophagy in chondrocytes. 
Studies have shown that resveratrol increases SIRT1, promoting cellular survival and decreasing inflammation and oxidative stress $^{(22,35-40)}$. SIRT1 enhances cellular survival by deacetylating LC3, which promotes autophagy(22) To investigate the role of SIRT1 in resveratrol autophagy reactivation, growth plates were evaluated for the presence of SIRT1 in untreated MTCOMP mice and those treated from birth to 4 weeks of age with resveratrol. SIRT1 was suppressed in MT-COMP growth plate chondrocytes (Fig. 1 Panel 30) and resveratrol treatment reverses the mutant COMP suppression of SIRT1 (Fig. 1 Panel 3P, N). These results demonstrate that resveratrol treatment promotes autophagy through SIRT1 in MT-COMP mice.

\section{Resveratrol modulates mTORC1 pathway signaling components}

We have previously shown that AKT is activated by MID1 and suppressed by PP2A in the mutant COMP pathology(4). AKT, an upstream regulator of $\mathrm{mTORC1}$, is up-regulated by PI3K$\mathrm{I}^{(41,42)}$. These studies evaluated whether PI3K-I elevates mTORC1 in MT-COMP growth plate chondrocytes in mice treated from birth to 4 weeks $^{(4)}$. Shown in Figure 2 Panel 1, PI3K-I was absent in untreated MT-COMP mice compared to controls. The absence of PI3K-1 in untreated MT-COMP growth plate chondrocytes in which mTORC1 signaling is high ${ }^{(4)}$ indicates that PI3K-I does not play a role in elevating mTORC1 signaling and blocking autophagy in MT-COMP growth plate pathology.

\section{Metformin does not activate autophagy clearance of MT-COMP}

Metformin modulates mTORC1 signaling by activating adenosine monophosphate-activated protein kinase $(\mathrm{AMPK})^{(43)}$, which in turn dampens mTORC1 signaling stimulating autophagy ${ }^{(42,44)}$. Previously, we showed that elevated mTORC1 signaling results from TNFa/TRAIL inflammation and ER stress ${ }^{(4)}$. We asked whether metformin treatment could induce autophagy in MT-COMP mice, thereby mimicking resveratrol's mechanism of action. Here, we assessed whether mTORC1 signaling could be reduced by metformin-stimulated AMPK activation in growth plate 
chondrocytes. As shown in Fig. 2 Panel 2, metformin administered to MT-COMP mice from birth to 4 weeks increased AMPK levels as expected, indicating that metformin reached the growth plate in therapeutic concentrations. However, metformin did not decrease either mutant COMP retention or pS6, which is a measure of mTORC1 signaling. These results demonstrate that metformin did not repress mTORC1 signaling (as measured by pS6) or induce autophagy in MTCOMP mice indicating that metformin does not mimic resveratrol's mechanism of action.

\section{Resveratrol postnatal treatment windows}

Previously, we demonstrated that resveratrol treatment in MT-COMP mice from birth to 4 weeks of age dampens mutant COMP intracellular ER retention, inflammation, chondrocyte death, and restored chondrocyte proliferation, resulting in increased femoral length ${ }^{(45)}$. To define the treatment windows in which resveratrol could be administered to MT-COMP mice to reduce chondrocyte pathology, resveratrol was started at birth, 1, 2 or 3 weeks of age and growth plates were collected at 4 weeks. As shown in Figure 3, resveratrol treatment for 1 - 3 weeks diminished intracellular retention, inflammation and chondrocyte cell death. The most dramatic reduction of inflammatory markers, interleukin 16 (IL-16) and YM1 (YM1 also known as eosinophil chemotactic factor-lymphocyte (ECF-L)), was observed in growth plates treated for three or more weeks with resveratrol (Fig. 3G-R). Importantly, resveratrol treatment beginning as late as 3 weeks of age reduced inflammation and chondrocyte death (Fig. 3I, O). Decreased cell death (TUNEL positive chondrocytes) showed a significant temporal response with treatment (Fig. 3A-F; M; EE). In contrast, chondrocyte proliferation did not recover until 3 weeks of resveratrol treatment (Fig. 3YDD; FF). Importantly, the timing of autophagy derepression (Fig. 3EE-JJ pS6) correlated with recovery of chondrocyte proliferation in response to resveratrol treatment suggesting that appropriate autophagy function plays a role in resveratrol's therapeutic action and growth plate chondrocyte cellular health. 


\section{Discussion}

The results of these studies establish that obstruction of autophagy causes mutant COMP accumulation in the ER of growth plate chondrocytes and, importantly, resveratrol clears and/or prevents intracellular retention by reactivating and/or maintaining autophagy function. The mechanism underlying this COMPopathy is the culmination of many experimentiments ${ }^{(3,5,46)}$, is caused by the retention of mutant COMP in the ER, which stimulates a chronic self-perpetuating pathological loop between ER stress, inflammation and oxidative that elevates mTORC1 signaling through a complex network involving $\mathrm{CHOP}$ and TNFa( ${ }^{(4)}$ (Fig. 4A). This this pathology can be interrupted by the loss of $\mathrm{CHOP}^{(5,46)}$ demonstrating that $\mathrm{CHOP}$ is an important therapeutic target. Resveratrol's multifocal targeted mechanisms of action is shown in Figure 4B includes the repression of $\mathrm{CHOP}$.

Our previous studies showed that resveratrol and aspirin dramatically reduce intracellular mutant COMP in the ER of MT-COMP growth plate chondrocytes, but the mechanism(s) is unknown ${ }^{(3)}$. In vitro and in vivo approaches were used to identify and validate the mechanism by which resveratrol reduces the ER intracellular retention. RCS cells expressing either mutant or wildtype COMP showed that autophagy was increased when treated with resveratrol (Fig. 2) and rapamycin treatment, an autophagy-inducing drug ${ }^{(47)}$, decreased the intracellular mutant COMP accumulation in growth plate chondrocytes in MT-COMP mice (Supplementary Fig. 2). Furthermore, pharmacologically blocking autophagy inhibited resveratrol clearance of mutant COMP demonstrating that resveratrol stimulated autophagy is the key molecular mechanism that clears mutant COMP preserving chondrocyte function and preventing cell death in MT-COMP mice (Fig. 1). These findings are consistent with previous studies in other cell types which showed that autophagy is the primary mechanism utilized to clear protein aggregates from the ER (48).

Autophagy plays an essential role in cellular maintenance by recycling cellular contents and participating in ER protein quality control(49). Many storage disorders involve a failure of or 
inefficient autophagy including proteinopathies (Alzheimer disease, tauopathies, Parkinson disease, Huntington disease), lipid storage disorders (Niemann Pick type C disease, Gaucher disease, Fabry disease) and glycogen storage disorders (Lafora disease, Von Gierke disease, Pompe disease $)^{(50,51)}$. Autophagy failure in disease occurs through a variety of mechanisms including impaired autophagosome formation, compromised autophagosome maturation (through mTOR activation, AMPK inhibition, SIRT1 downregulation) and improper recognition of autophagic cargo(51). In PSACH, a COMPopathy/proteinopathy, mTORC1 activation and SIRT1 downregulation blocks autophagosome maturation through a complex network (Figs. 1 and 4)(4). Chronic inflammation has been shown to reduce SIRT1 expression ${ }^{(52-56)}$ and the persistent inflammation in MT-COMP growth plate chondrocytes is likely to account for SIRT1 repression in MT-COMP mice (3). Recently, we showed that ER stress and TNFa/TRAIL upregulated MID1 and MID1 increased mTORC1 signaling directly and by decreasing PP2A and increasing phosphoAKT ${ }^{(4)}$. TNFa/TRAIL upregulation of MID1 has only been observed in airway inflammation and fibrosis ${ }^{(57-60)}$. This link between ER stress and MID1 is novel to the mutant COMP pathology ${ }^{(4)}$ (Fig. 4A). In contrast, PI3K-I and AMPK do not play a role in elevation of mTORC1 signaling in mutant COMP growth plate chondrocyte pathology (Fig. 2), whereas, these molecules have been shown to play a role in neurodegenerative diseases(61), obesity, inflammation, diabetes and cancer ${ }^{(62)}$. These findings show that not all of the mechanisms regulating autophagy in other diseases are involved in the mutant COMP induced autophagy block in growth plate chondrocytes. Specific to this model system mechanism, increased mTORC1 signaling and suppression of SIRT1 reduces autophagosome maturation causing the autophagy blockade. Treatment of MT-COMP mice with resveratrol reactivates autophagy and cellular proliferation, while repressing intracellular retention and inflammation (Fig. 3).

The pleotropic benefits from resveratrol, shown in the mechanistic model depicted in Fig. 4, makes it uniquely effective in addressing the multifaceted mutant COMP pathology. Resveratrol diminishes cellular stresses including inflammation, oxidative stress and blocked autophagy 
resulting in anti-aging, anti-cancer, neuro-protective, anti-diabetic and cardio-protective properties $^{(63-68)}$. Resveratrol promoted autophagy in the mutant COMP pathology by upregulating SIRT1 which increased the number of LC3 positive autophagosomes (Fig. 1 and 4B). Resveratrol also reduced TNFa which results in repressing mTORC1 signaling through decreased MID1, pAKT, pS6 and increased PP2A(4) (Fig. 4). Furthermore, treatment with resveratrol promotes autophagy by reducing oxidative stress and inflammation, which in turn alleviates ER stress ${ }^{(4)}$ (Fig. 4). These results demonstrate that resveratrol acts upon the mutant COMP pathology at multiple therapeutic points including ER stress, oxidative stress, inflammation (IL-1ß; IL-16; OSM; FCF-L/YM1; TNFa/TRAIL), autophagy, chondrocyte death and proliferation ultimately resulting in a $50 \%$ recovery of loss of long bone growth (Fig. 4$)^{(3,4)}$.

Although proteasomal degradation is not known to be the main mechanism clearing misfolded or aggregated protein from the ER, its role in a storage disease cannot be ruled out (48). Pharmacological blockade of proteasomal degradation along with resveratrol treatment resulted only in a minor decrease in clearance of mutant COMP (Fig. 1), indicating that proteasomal degradation is not the primary cellular mechanism by which resveratrol stimulates removal of mutant COMP from the ER of growth plate chondrocytes. Moreover, we demonstrated that retention of COMP in the ER allows for the assembly of an ordered matrix within ER vesicles of MT-COMP mice ${ }^{(7)}$. Once an intracellular matrix forms, there are no degradative enzymes to break up this large complex, which is likely too large to be translocated out of the ER to the proteasome for degradation ${ }^{(13)}$.

Importantly, the exciting findings from these studies show that there is a post-diagnosis window in which resveratrol diminishes growth plate pathology and clears mutant COMP from the ER in MT-COMP mice. Using our MT-COMP mouse that models PSACH clinical and molecular pathology in growth plate chondrocytes ${ }^{(7)}$, we showed that treatment with resveratrol from birth to adulthood successfully reduces murine growth plate pathology and restores some limb growth(3), indicating that resveratrol may be a therapeutic for PSACH. Early resveratrol treatment could be 
implemented in familial cases where diagnosis of PSACH can be made at birth. However, $80 \%$ of PSACH cases occur de novo, therefore, diagnosis occurs later, between 18- 24 months, when linear growth failure is recognized(69,70). Here, we asked whether resveratrol treatment postdiagnosis would be effective for de novo cases. Resveratrol treatment beginning from birth or 1 week of age had the best outcomes, substantially reducing COMP retention, inflammation and cell death and initiating the recovery of DNA proliferation in growth plate chondrocytes. Approximating from human to mouse growth (1 week of mouse growth is roughly equivalent to 2 years in human growth $\left.{ }^{(71)}\right)$, our findings suggest that resveratrol treatment at or shortly after diagnosis will likely provide some reduction of clinical and growth plate pathology. Importantly, 3 weeks of resveratrol treatment (treatment from $1-4$ wks) in the MT-COMP mouse increased autophagy (reduced pS6/mTORC1 signaling) demonstrating that autophagy reactivation is linked to the recovery of chondrocyte proliferation which most likely accounts for the recovery of some limb growth with resveratrol treatment. Interestingly, treatment beginning as late as 3 weeks of age (treatment from 3 - 4 wks) reduced inflammation and, therefore, would be expected to decrease joint pain suggesting even late treatment may have some benefit. The results for aspirin were similar to those with resveratrol with the exception that at least 2 weeks of aspirin treatment was required to reduce intracellular MT-COMP (Supplementary Figs. 1 and 3). Although aspirin reduced much of the mutant COMP growth plate pathology, aspirin is not approved for use in children under age 3 and caution must be used when administering aspirin to children/teens due to its association with Reyes syndrome (https://www.mayoclinic.org/diseases-conditions/reyessyndrome/symptoms-causes/syc-20377255). Importantly, resveratrol was more effective than aspirin at reducing mutant COMP ER accumulation most likely related to its effect on multiple molecules that stimulate autophagy and reduce inflammation and oxidative stress (Fig. 4). A combination of aspirin and resveratrol treatment was not superior to resveratrol therapy alone in MT-COMP mice suggesting that aspirin and resveratrol mechanisms of action overlap (data not shown). 
The outcome of these studies have important therapeutic applications because they show there is a therapeutic window during juvenile mouse growth in which resveratrol treatment diminished the mutant COMP chondrocyte pathology. This is a significant finding since most PSACH cases are not evident at birth. The absence of linear growth prompts diagnostic testing in children leading to PSACH diagnosis by 2 years of age. Moreover, these findings have important implications for addressing PSACH childhood pain that has not previously been appreciated or systematically treated $(3,5,16,72,73)$. Resveratrol's therapeutic use in PSACH children will be tested in future clinical trials. Currently, a phase 1 clinical trial is underway to assess the efficacy of resveratrol and its potential to relieve adult PSACH joint pain (NCT03866200 clinicaltrials.gov), although, earlier treatment would likely be more effective based on the findings presented here.

Resveratrol treatment is promising given that the potential therapeutic window spans the postdiagnosis period in PSACH and resveratrol decreases multiple cellular stresses, stimulates autophagy clearance of MT-COMP allowing chondrocytes to function and proliferate potentially increasing long bone growth. Collectively, these findings suggest that chondrocyte death, inflammation and pain are likely to improve prior to recovery of growth with resveratrol therapy and earlier treatment results in the best outcome.

\section{Acknowledgments}

We thank Frankie Chiu for technical assistance, dedication and organization. The authors have no conflict of interest to declare.

Funding: National Institute of Arthritis and Musculoskeletal and Skin Diseases of National Institutes of Health (RO1-AR057117-05) and the Leah Lewis Family Foundation. The content is solely the responsibility of the authors and does not necessarily represent the official views of the National Institutes of Health. 


\section{References}

1. Mortier GR, Cohn DH, Cormier-Daire V, Hall C, Krakow D, Mundlos S, et al. Nosology and classification of genetic skeletal disorders: 2019 revision. Am J Med Genet A. Dec 2019;179(12):2393-419. Epub 2019/10/22.

2. Hecht JT, Nelson LD, Crowder E, Wang Y, Elder FF, Harrison WR, et al. Mutations in exon 17B of cartilage oligomeric matrix protein (COMP) cause pseudoachondroplasia. Nat Genet. Jul 1995;10(3):325-9.

3. Posey KL, Coustry F, Veerisetty AC, Hossain M, Alcorn JL, Hecht JT. Antioxidant and anti-inflammatory agents mitigate pathology in a mouse model of pseudoachondroplasia. Hum Mol Genet. Jul 15 2015;24(14):3918-28.

4. Posey KL, Coustry F, Veerisetty AC, Hossain MG, Gambello MJ, Hecht JT. Novel mTORC1 Mechanism Suggests Therapeutic Targets for COMPopathies. Am J Pathol. Jan 2019;189(1):132-46. Epub 2018/12/17.

5. Posey KL, Coustry F, Veerisetty AC, Liu P, Alcorn JL, Hecht JT. Chop (Ddit3) is essential for D469del-COMP retention and cell death in chondrocytes in an inducible transgenic mouse model of pseudoachondroplasia. Am J Pathol. Feb 2012;180(2):727-37.

6. Posey KL, Coustry F, Veerisetty AC, Liu P, Alcorn JL, Hecht JT. Chondrocyte-specific pathology during skeletal growth and therapeutics in a murine model of pseudoachondroplasia. J Bone Miner Res. 2014;29(5):1258-68.

7. Posey KL, Veerisetty AC, Liu P, Wang HR, Poindexter BJ, Bick R, et al. An Inducible Cartilage Oligomeric Matrix Protein Mouse Model Recapitulates Human Pseudoachondroplasia Phenotype. The American Journal of Pathology. 2009/10 2009;175(4):1555-63.

8. Cooper RR, Ponseti IV, Maynard JA. Pseudoachondroplasia dwarfism. A rough-surfaced endoplasmic reticulum disorder. J Bone Joint Surg Am. 1973;55A:475-84. 
9. Cooper RR, Ponseti IV, Maynard JA. Pseudoachondroplastic dwarfism. A rough-surfaced endoplasmic reticulum storage disorder. Journal of Bone \& Joint Surgery - American Volume. 1973;55(3):475-84.

10. Briggs MD, Hoffman SM, King LM, Olsen AS, Mohrenweiser H, Leroy JG, et al. Pseudoachondroplasia and multiple epiphyseal dysplasia due to mutations in the cartilage oligomeric matrix protein gene. Nature Genetics. 1995;10(3):330-6.

11. Hecht JT, Nelson LD, Crowder E, Wang Y, Elder FFB, Harrison WR, et al. Mutations in exon 17B of cartilage oligomeric matrix protein (COMP) cause pseudoachondroplasia. Nature Genetics. 1995/07 1995;10(3):325-9.

12. Merritt TM, Bick R, Poindexter BJ, Alcorn JL, Hecht JT. Unique Matrix Structure in the Rough Endoplasmic Reticulum Cisternae of Pseudoachondroplasia Chondrocytes. The American Journal of Pathology. 2007/01 2007;170(1):293-300.

13. Posey KL, Hecht JT. Novel therapeutic interventions for pseudoachondroplasia. Bone. Mar 212017.

14. Coustry F, Posey KL, Liu P, Alcorn JL, Hecht JT. D469del-COMP retention in chondrocytes stimulates caspase-independent necroptosis. The American journal of pathology. Feb 2012;180(2):738-48.

15. Ciechanover A, Kwon YT. Degradation of misfolded proteins in neurodegenerative diseases: therapeutic targets and strategies. Exp Mol Med. Mar 13 2015;47:e147.

16. Posey KL, Alcorn JL, Hecht JT. Pseudoachondroplasia/COMP - translating from the bench to the bedside. Matrix Biol. Jul 2014;37:167-73.

17. Posey KL, Veerisetty AC, Liu P, Wang HR, Poindexter BJ, Bick R, et al. An inducible cartilage oligomeric matrix protein mouse model recapitulates human pseudoachondroplasia phenotype. Am J Pathol. Research Support, N.I.H., Extramural Research Support, Non-U.S. Gov't Oct 2009;175(4):1555-63. Epub 2009/09/19. 
18. Posey KL, Coustry F, Veerisetty AC, Liu P, Alcorn JL, Hecht JT. Chop (Ddit3) is essential for D469del-COMP retention and cell death in chondrocytes in an inducible transgenic mouse model of pseudoachondroplasia. Am J Pathol. Research Support, N.I.H., Extramural

Research Support, Non-U.S. Gov't Feb 2012;180(2):727-37. Epub 2011/12/14.

19. Marciniak SJ, Yun CY, Oyadomari S, Novoa I, Zhang Y, Jungreis R, et al. CHOP induces death by promoting protein synthesis and oxidation in the stressed endoplasmic reticulum. Genes Dev. Dec 15 2004;18(24):3066-77.

20. Kulkarni SS, Canto C. The molecular targets of resveratrol. Biochim Biophys Acta. Jun 2015;1852(6):1114-23.

21. Schmatz R, Perreira LB, Stefanello N, Mazzanti C, Spanevello R, Gutierres J, et al. Effects of resveratrol on biomarkers of oxidative stress and on the activity of delta aminolevulinic acid dehydratase in liver and kidney of streptozotocin-induced diabetic rats. Biochimie. Research Support, Non-U.S. Gov't Feb 2012;94(2):374-83. Epub 2011/08/26.

22. Tennen RI, Michishita-Kioi E, Chua KF. Finding a target for resveratrol. Cell. Feb 3 2012;148(3):387-9. Epub 2012/02/07.

23. Wacheck V. mTOR pathway inhibitors in cancer therapy: moving past rapamycin. Pharmacogenomics. Comparative Study

Editorial Sep 2010;11(9):1189-91. Epub 2010/09/24.

24. Way SW, Rozas NS, Wu HC, McKenna J, 3rd, Reith RM, Hashmi SS, et al. The differential effects of prenatal and/or postnatal rapamycin on neurodevelopmental defects and cognition in a neuroglial mouse model of tuberous sclerosis complex. Hum Mol Genet. Comparative Study

Research Support, N.I.H., Extramural

Research Support, Non-U.S. Gov't Jul 15 2012;21(14):3226-36. Epub 2012/04/26. 
25. Burkewitz K, Weir HJ, Mair WB. AMPK as a Pro-longevity Target. Exp Suppl. 2016;107:227-56. Epub 2016/11/05.

26. Lushchak O, Strilbytska O, Piskovatska V, Storey KB, Koliada A, Vaiserman A. The role of the TOR pathway in mediating the link between nutrition and longevity. Mech Ageing Dev. Jun 2017;164:127-38. Epub 2017/03/21.

27. Lien F, Berthier A, Bouchaert E, Gheeraert C, Alexandre J, Porez G, et al. Metformin interferes with bile acid homeostasis through AMPK-FXR crosstalk. J Clin Invest. Research Support, Non-U.S. Gov't Mar 2014;124(3):1037-51. Epub 2014/02/18.

28. Mauthe M, Orhon I, Rocchi C, Zhou X, Luhr M, Hijlkema KJ, et al. Chloroquine inhibits autophagic flux by decreasing autophagosome-lysosome fusion. Autophagy. 2018;14(8):1435-55.

29. Yuan N, Song L, Zhang S, Lin W, Cao Y, Xu F, et al. Bafilomycin A1 targets both autophagy and apoptosis pathways in pediatric B-cell acute lymphoblastic leukemia. Haematologica. Mar 2015;100(3):345-56.

30. Adams J. The proteasome: structure, function, and role in the cell. Cancer Treat Rev. May 2003;29 Suppl 1:3-9. Epub 2003/05/10.

31. Eriksson E, Zaman F, Chrysis D, Wehtje H, Heino TJ, Savendahl L. Bortezomib is cytotoxic to the human growth plate and permanently impairs bone growth in young mice. PLoS One. 2012;7(11):e50523.

32. Hu LH, Fan YJ, Li Q, Guan JM, Qu B, Pei FH, et al. Bortezomib protects against dextran sulfate sodiuminduced ulcerative colitis in mice. Mol Med Rep. Jun 2017;15(6):4093-9.

33. Kadowaki $\mathrm{H}$, Nishitoh $\mathrm{H}$. Endoplasmic reticulum quality control by garbage disposal. FEBS J. Jan 2019;286(2):232-40. Epub 2018/06/21.

34. Almonte-Becerril M, Navarro-Garcia F, Gonzalez-Robles A, Vega-Lopez MA, Lavalle C, Kouri JB. Cell death of chondrocytes is a combination between apoptosis and autophagy 
during the pathogenesis of Osteoarthritis within an experimental model. Apoptosis. May 2010;15(5):631-8.

35. Guo W, Qian L, Zhang J, Zhang W, Morrison A, Hayes P, et al. Sirt1 overexpression in neurons promotes neurite outgrowth and cell survival through inhibition of the mTOR signaling. J Neurosci Res. Nov 2011;89(11):1723-36.

36. Albani D, Polito L, Batelli S, De Mauro S, Fracasso C, Martelli G, et al. The SIRT1 activator resveratrol protects SK-N-BE cells from oxidative stress and against toxicity caused by alpha-synuclein or amyloid-beta (1-42) peptide. Journal of Neurochemistry. Comparative Study

Research Support, Non-U.S. Gov't Sep 2009;110(5):1445-56. Epub 2009/06/30.

37. Csiszar A, Labinskyy N, Podlutsky A, Kaminski PM, Wolin MS, Zhang C, et al. Vasoprotective effects of resveratrol and SIRT1: attenuation of cigarette smoke-induced oxidative stress and proinflammatory phenotypic alterations. Am J Physiol Heart Circ Physiol. Research Support, N.I.H., Extramural

Research Support, N.I.H., Intramural

Research Support, Non-U.S. Gov't Jun 2008;294(6):H2721-35. Epub 2008/04/22.

38. Guo H, Chen Y, Liao L, Wu W. Resveratrol protects HUVECs from oxidized-LDL induced oxidative damage by autophagy upregulation via the AMPK/SIRT1 pathway. Cardiovascular Drugs and Therapy. Research Support, Non-U.S. Gov't Jun 2013;27(3):189-98. Epub 2013/01/30.

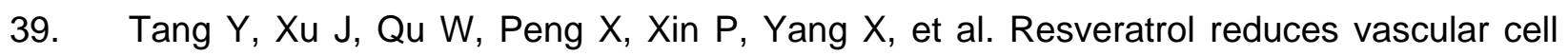
senescence through attenuation of oxidative stress by SIRT1/NADPH oxidase-dependent mechanisms. J Nutr Biochem. Research Support, Non-U.S. Gov't Nov 2012;23(11):14106. Epub 2012/01/31. 
40. Yun JM, Chien A, Jialal I, Devaraj S. Resveratrol up-regulates SIRT1 and inhibits cellular oxidative stress in the diabetic milieu: mechanistic insights. J Nutr Biochem. Jul 2012;23(7):699-705. Epub 2011/08/05.

41. Hennessy BT, Smith DL, Ram PT, Lu Y, Mills GB. Exploiting the PI3K/AKT pathway for cancer drug discovery. Nat Rev Drug Discov. Dec 2005;4(12):988-1004. Epub 2005/12/13.

42. Herschbein L, Liesveld JL. Dueling for dual inhibition: Means to enhance effectiveness of PI3K/Akt/mTOR inhibitors in AML. Blood Rev. May 2018;32(3):235-48. Epub 2017/12/26.

43. Chen $\mathrm{H}$, Vanhoutte PM, Leung SWS. Vascular adenosine monophosphate-activated protein kinase: Enhancer, brake or both? Basic Clin Pharmacol Toxicol. Oct 312019. Epub 2019/11/02.

44. Kim J, You YJ. Regulation of organelle function by metformin. IUBMB Life. Jul 2017;69(7):459-69.

45. Posey KL, Coustry F, Veerisetty AC, Hossain M, Gattis D, Booten S, et al. Antisense Reduction of Mutant COMP Reduces Growth Plate Chondrocyte Pathology. Mol Ther. Mar 01 2017;25(3):705-14.

46. Coustry F, Posey KL, Liu P, Alcorn JL, Hecht JT. D469del-COMP Retention in Chondrocytes Stimulates Caspase-Independent Necroptosis. The American Journal of Pathology. 2012/02 2012;180(2):738-48.

47. Nalbandian A, Llewellyn KJ, Nguyen C, Yazdi PG, Kimonis VE. Rapamycin and chloroquine: the in vitro and in vivo effects of autophagy-modifying drugs show promising results in valosin containing protein multisystem proteinopathy. PLoS One. 2015;10(4):e0122888.

48. Kocaturk NM, Gozuacik D. Crosstalk Between Mammalian Autophagy and the UbiquitinProteasome System. Front Cell Dev Biol. 2018;6:128. Epub 2018/10/20. 
49. Chino H, Mizushima N. ER-Phagy: Quality Control and Turnover of Endoplasmic Reticulum. Trends Cell Biol. May 2020;30(5):384-98. Epub 2020/04/18.

50. Ramesh J, Ronsard L, Gao A, Venugopal B. Autophagy Intertwines with Different Diseases-Recent Strategies for Therapeutic Approaches. Diseases. Feb 1 2019;7(1). Epub 2019/02/06.

51. Palhegyi AM, Seranova E, Dimova S, Hoque S, Sarkar S. Biomedical Implications of Autophagy in Macromolecule Storage Disorders. Front Cell Dev Biol. 2019;7:179. Epub $2019 / 09 / 27$.

52. Liu TF, McCall CE. Deacetylation by SIRT1 Reprograms Inflammation and Cancer. Genes Cancer. Mar 2013;4(3-4):135-47. Epub 2013/09/11.

53. Yoshino J, Mills KF, Yoon MJ, Imai S. Nicotinamide mononucleotide, a key NAD(+) intermediate, treats the pathophysiology of diet- and age-induced diabetes in mice. Cell Metab. Oct 5 2011;14(4):528-36. Epub 2011/10/11.

54. Kim HJ, Kim JH, Noh S, Hur HJ, Sung MJ, Hwang JT, et al. Metabolomic analysis of livers and serum from high-fat diet induced obese mice. J Proteome Res. Feb 4 2011;10(2):72231. Epub 2010/11/05.

55. Kendrick AA, Choudhury M, Rahman SM, McCurdy CE, Friederich M, Van Hove JL, et al. Fatty liver is associated with reduced SIRT3 activity and mitochondrial protein hyperacetylation. Biochem J. Feb 1 2011;433(3):505-14. Epub 2010/11/04.

56. Tao R, Wei D, Gao H, Liu Y, DePinho RA, Dong XC. Hepatic FoxOs regulate lipid metabolism via modulation of expression of the nicotinamide phosphoribosyltransferase gene. J Biol Chem. Apr 22 2011;286(16):14681-90. Epub 2011/03/11.

57. Collison A, Li J, Pereira de Siqueira A, Zhang J, Toop HD, Morris JC, et al. Tumor necrosis factor-related apoptosis-inducing ligand regulates hallmark features of airways remodeling in allergic airways disease. Am J Respir Cell Mol Biol. Jul 2014;51(1):86-93. 
58. Collison AM, Sokulsky LA, Sherrill JD, Nightingale S, Hatchwell L, Talley NJ, et al. TNFrelated apoptosis-inducing ligand (TRAIL) regulates midline-1, thymic stromal lymphopoietin, inflammation, and remodeling in experimental eosinophilic esophagitis. J Allergy Clin Immunol. Oct 2015;136(4):971-82.

59. Sokulsky LA, Collison AM, Nightingale S, Fevre AL, Percival E, Starkey MR, et al. TRAIL deficiency and PP2A activation with salmeterol ameliorates egg allergen-driven eosinophilic esophagitis. Am J Physiol Gastrointest Liver Physiol. Dec 1 2016;311(6):G998-G1008. Epub 2016/10/16.

60. Collison AM, Li J, de Siqueira AP, Lv X, Toop HD, Morris JC, et al. TRAIL signals through the ubiquitin ligase MID1 to promote pulmonary fibrosis. BMC Pulm Med. Feb 7 2019;19(1):31. Epub 2019/02/09.

61. $\mathrm{Xu} \mathrm{F}, \mathrm{Na} \mathrm{L}$, Li Y, Chen L. Roles of the PI3K/AKT/mTOR signalling pathways in neurodegenerative diseases and tumours. Cell Biosci. 2020;10:54. Epub 2020/04/09.

62. Jeon SM. Regulation and function of AMPK in physiology and diseases. Exp Mol Med. Jul 15 2016;48(7):e245. Epub 2016/07/16.

63. Park D, Jeong H, Lee MN, Koh A, Kwon O, Yang YR, et al. Resveratrol induces autophagy by directly inhibiting mTOR through ATP competition. Sci Rep. Feb 23 2016;6:21772. Epub 2016/02/24.

64. Lee J, Hong SW, Kwon H, Park SE, Rhee EJ, Park CY, et al. Resveratrol, an activator of SIRT1, improves ER stress by increasing clusterin expression in HepG2 cells. Cell Stress Chaperones. Jul 2019;24(4):825-33. Epub 2019/06/12.

65. Armour SM, Baur JA, Hsieh SN, Land-Bracha A, Thomas SM, Sinclair DA. Inhibition of mammalian S6 kinase by resveratrol suppresses autophagy. Aging (Albany NY). Jun 3 2009;1(6):515-28. Epub 2010/02/17. 
66. Wang N, He J, Pan C, Wang J, Ma M, Shi X, et al. Resveratrol Activates Autophagy via the AKT/mTOR Signaling Pathway to Improve Cognitive Dysfunction in Rats With Chronic Cerebral Hypoperfusion. Front Neurosci. 2019;13:859. Epub 2019/09/05.

67. Bonnefont-Rousselot D. Resveratrol and Cardiovascular Diseases. Nutrients. May 2 2016;8(5). Epub 2016/05/06.

68. Nunes S, Danesi F, Del Rio D, Silva P. Resveratrol and inflammatory bowel disease: the evidence so far. Nutr Res Rev. Jun 2018;31(1):85-97. Epub 2017/12/02.

69. McKeand J, Rotta J, Hecht JT. Natural history study of pseudoachondroplasia. Am J Med Genet. May 17 1996;63(2):406-10.

70. Unger S, Hecht JT. Pseudoachondroplasia and multiple epiphyseal dysplasia: New etiologic developments. Am J Med Genet. Winter 2001;106(4):244-50.

71. Frindik JP, Baptista J. Adult height in growth hormone deficiency: historical perspective and examples from the national cooperative growth study. Pediatrics. Oct 1999;104(4 Pt 2):1000-4. Epub 1999/10/03.

72. Gamble C, Nguyen J, Hashmi SS, Hecht JT. Pseudoachondroplasia and painful sequelae. Am J Med Genet A. Nov 2015;167(11):2618-22.

73. Posey KL, Coustry F, Hecht JT. Cartilage oligomeric matrix protein: COMPopathies and beyond. Matrix Biol. Oct 2018;71-72:161-73. Epub 2018/03/14. 
Figure legends

Figure 1. Reactivation of autophagy correlates with reduction in intracellular COMP retention. Panel 1: Growth plate sections from MT-COMP mice at age 4 weeks were immunostained with human COMP antibody after different treatments: A) no treatment, B) resveratrol treatment of $0.25 \mathrm{~g} / \mathrm{L}$ in drinking water from birth to 4 weeks, C) resveratrol and bafilomycin A (0.3 mg/Kg injected $5 X$ per week from 1-4 weeks) treatments or D) bortezomib $(0.1 \mathrm{mg} / \mathrm{Kg} 3 \mathrm{X}$ per week from 1-4 weeks). Bafilomycin A blocks the fusion of the autophagosome to the lysosome ${ }^{(28)}$, while Bortezomib is a proteasomal inhibitor ${ }^{(30)}$. Bafilomycin A blocks autophagy in resveratrol treated growth plates (C) while bortezomib only partially blocks resveratrol (D) clearance of MT-COMP. Each treatment group included 10 animals. Panel 2: RCS cells expressing either WT- or MT-COMP immunostained for LC3: untreated (E, I), resveratrol $(\mathbf{F}, \mathbf{J})$, chloroquine $(\mathbf{G}, \mathbf{K})$ and resveratrol and chloroquine $(\mathbf{H}, \mathbf{L})$ treatments. Quantification of LC3 positive foci (M) in MT- and WT-COMP RCS cells. Bars = means with standard deviation. Chloroquine blocks fusion between the lysosome and autophagasome (28). Res = resveratrol; ChIQ = chloroquine; MT-COMP = mutant COMP; WT-COMP = wild-type COMP; ${ }^{\star \star} \mathrm{P} \leq 0.005 ;{ }^{* \star *} \mathrm{P} \leq 0.0005$. LC3 foci were quantified in $30-50$ cells in 10 different images for each group. Panel 3: SIRT1 immunostaining of control (no treatment) (N), untreated MTCOMP (O) and resveratrol treated MT-COMP (P) growth plates are shown. Ten animals were included in each treatment group

Figure 2. PI3K-1 and AMPK do not play a role in mutant COMP mTORC1 signaling.

Panel 1 shows results of resveratrol and Panel 2 metformin treatments. Each treatment group contained 10 mice. Growth plates at 4 weeks: Control (A, D, G, J), MT-COMP untreated (B, E, $\mathbf{H}, \mathbf{K})$, resveratrol $(0.25 \mathrm{~g} / \mathrm{L}$ of resveratrol in drinking water from birth to 4 weeks) (C) or metformin $(0.5 \mathrm{mg} / \mathrm{ml}$ in drinking water from birth to 4 weeks) $(\mathbf{F}, \mathbf{I}, \mathbf{L})$. Immunostaining of PI3K-I (A-C), human COMP (D-F), pAMPK (G-I) and phospho-S6 (pS6) (J-L) is shown. Bars $=100 \mu \mathrm{m}$. 
Figure 3. Resveratrol treatment window. Treatments started at 1 or 2 or 3 weeks after birth and stopped at 4 weeks and evaluated for mutant COMP pathology. Ten mice were included in each treatment group. Treatments starting at birth or 1 week of age produced the best therapeutic outcomes $(\mathbf{K}, \mathbf{L})$. Later therapy dampened but did not eliminate the disease progression and did not restore DNA proliferation (C). Resveratrol treatment times are shown in the shaded boxes. Control (C57BLI6) (A, G, M, S, Y, EE) and MT-COMP with no treatment (B, H, N, T, Z, FF) resveratrol treatment for 1 (C, I, O, U, AA, GG), 2 (D, J, P, V, BB, HH), $3(\mathbf{E}, \mathbf{K}, \mathbf{Q}, \mathbf{W}, \mathbf{C C}$, II) or $4(\mathbf{F}, \mathbf{L}, \mathbf{R}, \mathbf{X}, \mathbf{D D}, \mathbf{J J})$ weeks and growth plates at 4 weeks were evaluated for human COMP retention, IL-16 and YM1, TUNEL (cell death), PCNA (cell proliferation) and mTORC1 signaling (pS6). Quantification of TUNEL positive chondrocytes for different treatment periods are shown in $\mathrm{KK}$ and summary of findings in LL. Bars in $\mathrm{KK}=$ means with standard deviation. Resveratrol treatment decreased intracellular MT-COMP (C) and IL-16 and YM1 inflammation (I, $\mathbf{0})$ after one week of treatment compared to untreated MT-COMP growth plates $(\mathbf{H}, \mathbf{N})$ and mTORC1 signaling was substantially reduced after 3 weeks of treatment $(\mathbf{I I}, \mathbf{J J})$. The relative change of MT-COMP pathology on treatment period is shown in LL.

Figure 4. Mechanisms by which resveratrol treatment inhibits MT-COMP growth plate chondrocyte pathology. A) Pathologic mechanism initiated and maintained by retention of MTCOMP in ER of growth plate chondrocytes of MT-COMP mice ${ }^{(3-5)}$. B) Resveratrol actions in pathologic processes that reduced chondrocyte ER stress including less inflammation and oxidative stress $^{(3)}$ (shown in orange) and decreases PAKT, MID1 and mTORC1 signaling which blocks autophagy $^{(4)}$ (green) through activation of SIRT1 that promote autophagosome maturation (shown in purple). 
Supplemental Figure 1: Aspirin treatment window. Treatments started at 1 or 2 or 3 weeks after birth and stopped at 4 weeks and evaluated for mutant COMP pathology. Each treatment group included 10 mice. Aspirin treatments are shown in the shaded boxes. Treatments starting at birth or 1 week of age produced the best therapeutic outcomes $(\mathbf{K}, \mathbf{L})$, later therapy mitigated but did not eliminate the disease progression (C). Control (C57BLI6) (A, G, M, S, Y, EE) and MTCOMP with no treatment $(\mathbf{B}, \mathbf{H}, \mathbf{N}, \mathbf{T}, \mathbf{Z}, \mathbf{F F})$ resveratrol treatment for $1(\mathbf{C}, \mathbf{I}, \mathbf{O}, \mathbf{U}, \mathbf{A A}, \mathbf{G G}), 2$ (D, J, P, V, BB, HH), 3 (E, K, Q, W, CC, II) or 4 (F, L, R, X, DD, JJ) weeks and growth plates at 4 weeks were evaluated for human COMP retention, IL-16 and YM1, TUNEL (cell death), PCNA and mTORC1 signaling. Quantification of TUNEL positive chondrocytes for different treatment periods are shown in KK. Bars = means with standard deviation. Aspirin treatment decreased intracellular MT-COMP after two weeks of treatment (D) and decreased in IL-16 and YM1 inflammation after one week of treatment $(\mathbf{I}, \mathbf{O})$ compared to $(\mathbf{H}, \mathbf{N})$. Aspirin decreased cell death after 2 weeks of treatment $(\mathbf{U})$, mTORC1 signaling after 3 weeks of treatment $(\mathbf{J J})$ and increased proliferation after 3 weeks of treatment (CC). The relative change of MT-COMP pathology on treatment period is shown in LL.

\section{Supplemental Figure 2. Rapamycin activates autophagy in MT-COMP growth plate chondrocytes.}

Rapamycin reduces MT-COMP retention in chondrocytes. Immunostaining with human COMP antibody of: A MT-COMP growth plate chondrocytes at 4 weeks with no treatment, B control vehicle, C rapamycin ( $1 \mathrm{mg} / \mathrm{kg} 5$ IP injections per week from 1 to 4 weeks of age). Ten mice were included in each treatment group. 
bioRxiv preprint doi: https://doi.org/10.1101/2020.10.26.355628; this version posted October 27, 2020. The copyright holder for this preprint (which was not certified by peer review) is the author/funder. All rights reserved. No reuse allowed without permission.
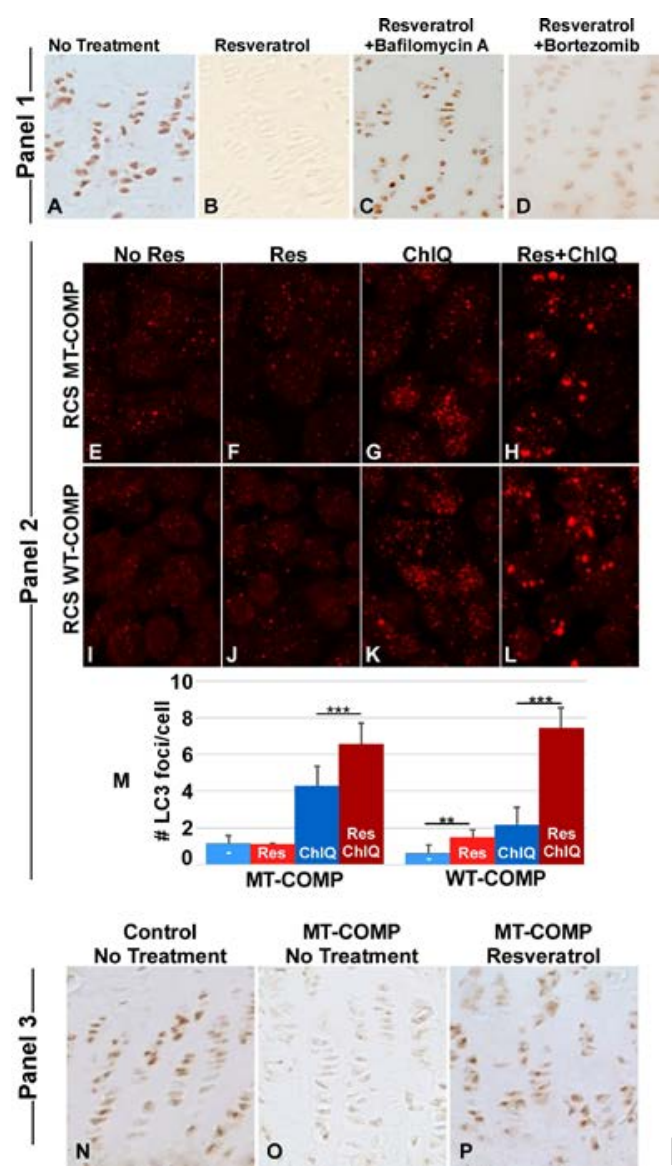

Fig. 1

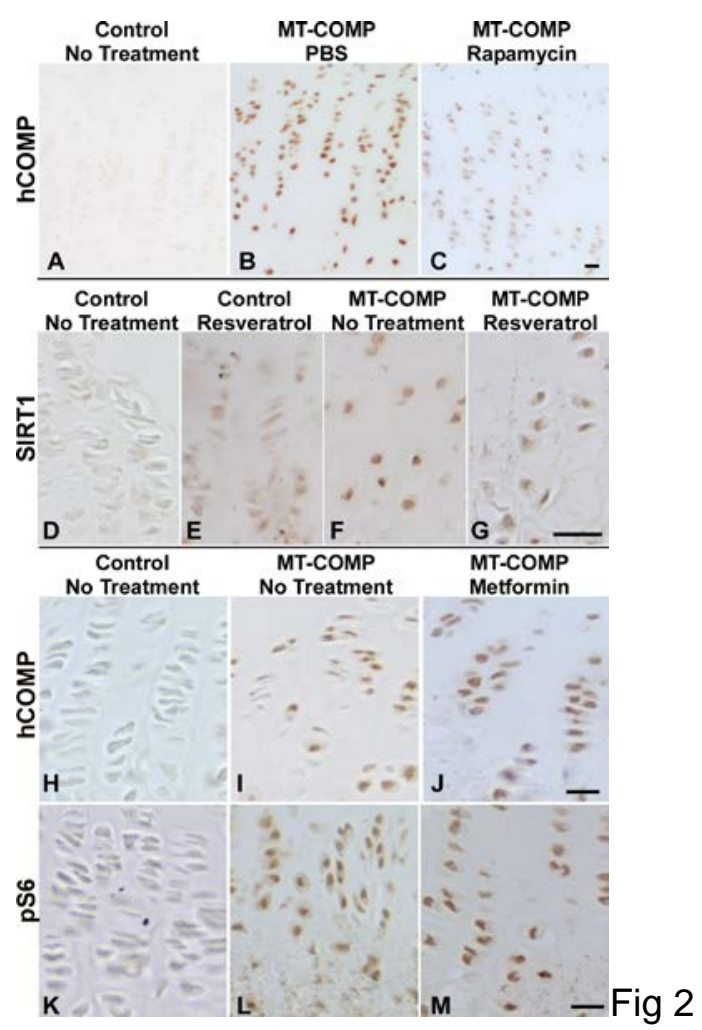




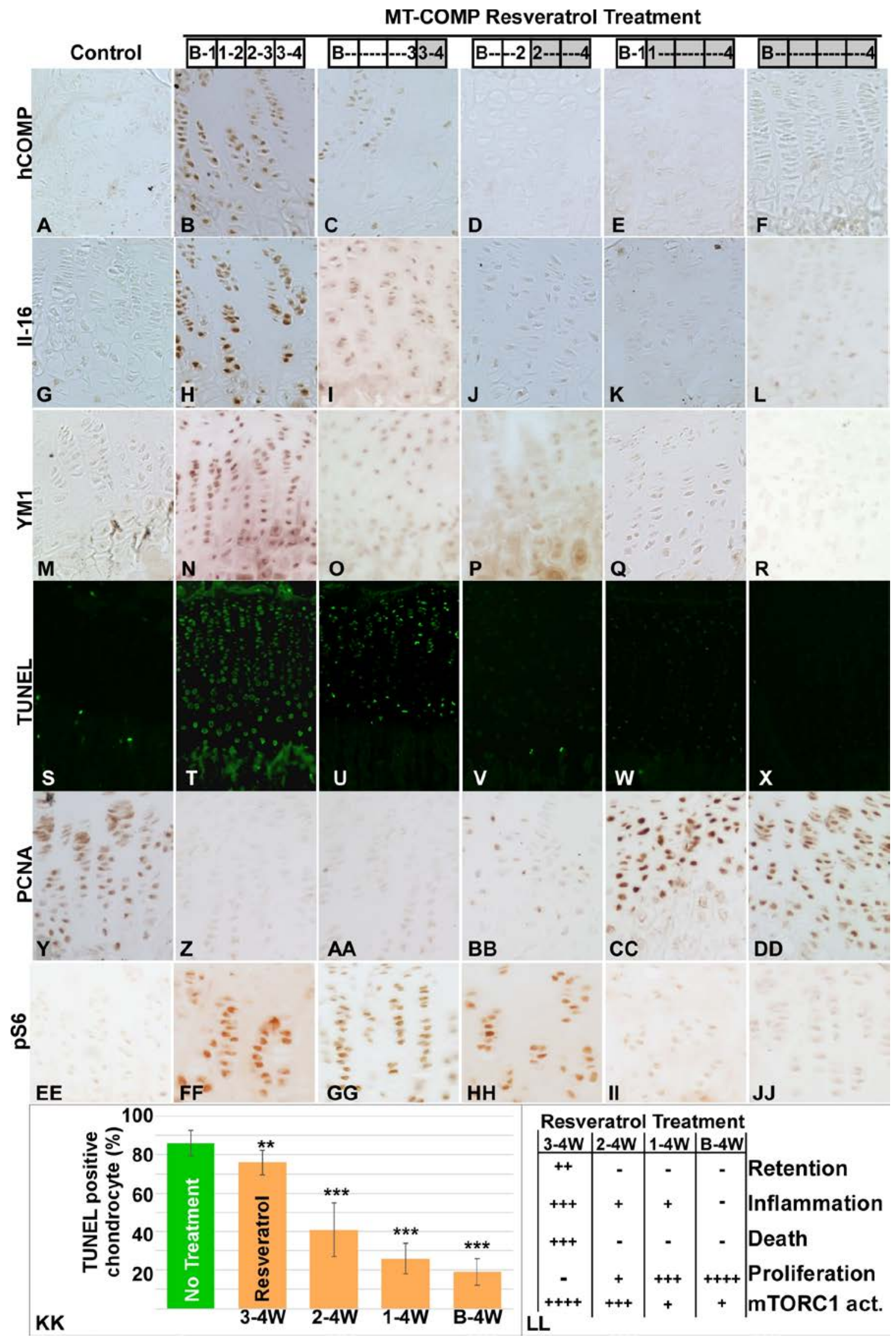

Fig. 3 
bioRxiv preprint doi: https://doi.org/10.1101/2020.10.26.355628; this version posted October 27, 2020. The copyright holder for this preprint (which was not certified by peer review) is the author/funder. All rights reserved. No reuse allowed without permission.

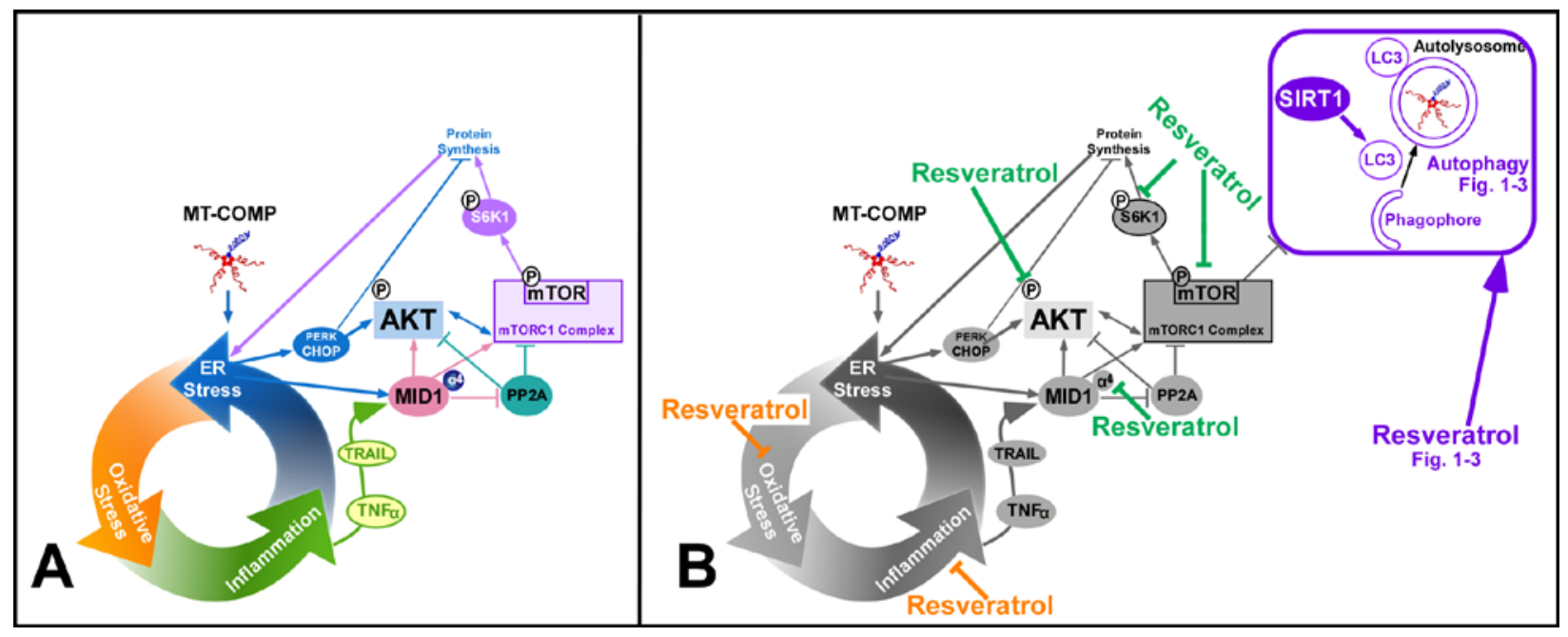

Fig. 4 




Fig. S1 
bioRxiv preprint doi: https://doi.org/10.1101/2020.10.26.355628; this version posted October 27, 2020. The copyright holder for this preprint (which was not certified by peer review) is the author/funder. All rights reserved. No reuse allowed without permission.

A

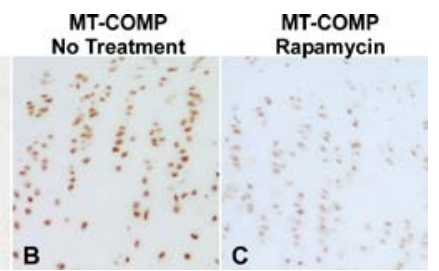

Fig. S2 